\title{
Gender and reproductive health services: Putting gender perspective into practice
}

Frontiers in Reproductive Health

Follow this and additional works at: https://knowledgecommons.popcouncil.org/departments_sbsr-rh

Part of the Demography, Population, and Ecology Commons, Family, Life Course, and Society Commons, Health Services Research Commons, International Public Health Commons, and the Medicine and Health Commons

How does access to this work benefit you? Let us know!

\section{Recommended Citation}

"Gender and reproductive health services: Putting gender perspective into practice," FRONTIERS Legacy Themes. Washington, DC: Population Council, 2009. 


\section{Population Council FRONTIERS \\ IN REPRODUCTIVE HEALTH}

\section{Legacy Series:}

\section{GENDER AND REPRODUCTIVE HEALTH SERVICES}

\section{Putting Gender Perspective into Practice}

Promotion of gender equity is widely acknowledged as important for improving reproductive health programs. Gender shapes all aspects of reproductive health $(\mathrm{RH})$ service delivery:

Whether clients can meet with a provider, in a clinic or community

$\checkmark$ What they can openly discuss with provider

$\checkmark$ What they can do with information received

$\checkmark$ Which services may be acceptable to partners

Thus, promoting gender equity-fairness and justice in responsibilities and access to benefits to women, men, girls, and boys-is a major goal for $\mathrm{RH}$ programs. But how can programs operationalize the concept of gender perspective when delivering family planning (FP) and other reproductive health (RH) services? What kind of impact does this have on use and effectiveness? FRONTIERS projects have improved understanding of the impact of gender issues on reproductive health, as well as helping identify effective actions for incorporating gender perspective into services.

\section{How to Be Certified "Gender-Sensitive"*}

1) Initial self-assessment using standardized tools

2) Development and implementation of improvement plans with own resources

3) Meet minimum of $80 \%$ of set of quality and gender standards

4) External assessment ensuring standards have been met

5) Certification at public meeting with plaque placed at clinic to inform clients of quality offered at unit

*Certification is valid for 2 years so process must be repeated 


\title{
Evidence-based Measures for Approaching Gender Equity
}

\author{
Incorporating Gender into Services
}

Certification of health facilities as "gender sensitive" is feasible and affordable. Organizations can introduce a gender perspective with their own resources and minimal assistance by following a proven certification strategy, in which a health facility needs to achieve quality of care and gender standards to be certified "gender-sensitive." The strategy includes a guidance tool with instructions helping facilities conduct self-assessments, identify strengths and deficiencies in terms of gender-sensitive services, and develop action plans to improve gender-related services (Palenque et al. 2004; Palenque, Riveros-Hamel, and Vernon 2007). Examples of concrete actions include: ensuring providers are familiar with concepts of gender and gender inequality; establishing explicit institutional policies to forbid genderbased discrimination; use of non-discriminatory language; establishing office hours around women's availability; and use of gender-sensitive IEC materials to empower women to make informed decisions. Guidelines for implementing certification strategy, which has now been introduced in at least four Latin American countries, are available in English and Spanish (Riveros-Hamel et al. 2008; Riveros-Hamel, Martin, and Vernon 2008).

\section{Introducing a gender perspective through certification decreases unmet need} for family planning among clients and improves communication and decision-making dynamics between couples. In those clinics in which the strategy was originally introduced, the proportion of married women of fertile age not wanting pregnancy in the following two years or did not want more children, and who were not using a contraceptive, although they would like to, decreased by nearly 35 percent as a result of the certification process (Palenque et al. 2004).

\section{Engaging Men in Safeguarding Reproductive Health}

\section{Joint counseling of women attending ANC services and their partners can lead} to improved couple communication and reproductive health benefits. In many developing countries, men are the major decision-makers on reproductive health but are poorly informed about or engaged in services to support partner health. In India and South Africa, couples were offered joint, individual, or same-sex group counseling sessions during antenatal care visits. Men demonstrate great interest in learning more about partners' needs, but the intervention had very different results in the two settings. 
In New Delhi, participating couples were married and members of the husband's employer-provided government health insurance scheme. When men were offered joint counseling sessions at the dispensary, twice as many husbands (28\%) attended sessions when not offered. Couples reported more communication on family planning ( $84 \%$ versus $64 \%$, respectively) and more joint decision-making on the issue ( $91 \%$ versus $71 \%$ ). Moreover,

this model led to significantly higher levels of postpartum contraception compared with couples in which women received regular ANC (Caleb-Varkey et al. 2004). These results have led to this model being scaled up from the original three dispensaries to all 30 facilities in the insurance scheme (Caleb-Varkey, Mishra, and Khan 2008).

In urban and rural clinics of KwaZulu Natal, South Africa, implementing joint counseling was challenging. Fewer than 10 percent of pregnant women were married and only one quarter lived with partners. One-third of couples invited did attend joint counseling, communication was reported to improve, and male partners were more likely to give support in pregnancy complications; however, postpartum family planning and overall risk behavior did not change (Kunene et al. 2004).

\section{Men will use RH services from clinics traditionally oriented to women.}

Integrating RH services for males into female-focused primary health care clinics increases number of both male and female clients and reduces cost per client. Findings from Bangladesh show the monthly average of male clients per clinic nearly tripled, from 131 to 345, and women's use of any service increased from average of 425 to 693 per clinic per month (Hossain et al. 2004; Rob et al. 2004). These improved and integrated services have been successfully scaled up to 40 additional clinics, with similar increases in utilization by both men and women (Mannan et al. 2008).

\section{Men are effective as community-based distribution (CBD) agents, particularly}

for reaching men. Numerous studies show recruiting men for community-based family planning services is feasible and effective. Studies in Cameroon, Ghana, Kenya, and Mali showed communities readily accept male CBD agents. Moreover, comparing condom sales by male and female agents showed men sold twice (Peru) or almost three times (Kenya) as many condoms as women. Studies in Latin America and Africa show male and female CBD agents can be equally productive overall in providing couple-years of protection (Foreit et al. 1992; Green, Foreit, and Joyce 2001; Sanogo et al. 2004). 


\section{Addressing Gender-based Violence}

Health providers can be trained to address domestic violence during regular $\mathbf{R H}$ services. After training 400 health providers in Mexico and Peru, follow-up interviews with a sample of 100 providers found 95 percent providing services, information, and counseling regarding domestic violence to clients, and almost half $(45 \%)$ reported training other providers. The study reinforced need for establishing institutions assisting domestic violence survivors, for providing continued support after experiences are identified during RH consultations (Pick and Miranda 1999).

\section{Post-rape care protocols and services remain poorly known or implemented.}

Many countries have developed policies and protocols on post-rape care, including emergency contraception and HIV prevention; implementation is, however, weak. A situation analysis in seven cities in Bangladesh and India showed no uniformity in service provision. Police had little or no training on managing or counseling rape survivors, and hospitals had few providers skilled in medical management of rape cases. Services for comprehensive management, including STI prophylaxis, referrals for HIV C\&T, and PEP, were little-known and rarely available (Khan et al. 2008).

\section{Post-rape care can be integrated into existing outpatient services at district} hospital level. An intervention in South Africa tested feasibility of creating a standardized and routine package of post-rape care services within the outpatient department of a district hospital, including establishing a sexual violence advisory committee within the hospital; developing and instituting a rape management policy; training healthcare workers and other providers in specific services; centralizing and coordinating post-rape care by designating a specific and private room in the outpatient department; and undertaking community awareness campaigns about service availability. The service necessitated fewer interactions with providers, quality of care substantially improved across all domains, patients were more likely to receive HIV PEP and complete the full 28-day regimen, and mean time interval reduced from 28 to 18 hours between assault and receiving first PEP dose. Importantly, this study demonstrated it is possible to substantially expand nurses' roles in sexual assault medical management (Kim et al. 2007).

\section{Medical management of post-rape care needs stronger linkages with the legal} system and the capacity to meet the needs of child survivors of both sexes. Despite demonstrating competence in medical care of rape survivors, most nurses were reluctant to learn and implement forensic examination, citing perceived inability and unwillingness to testify in court if needed. The South Africa study corroborated evidence from other countries, that one-fourth of survivors were less than 14, about half were under 18, and several were male. The package of care services had assumed a largely adult clientele, so adjustments need to be made, in policy and services, to ensure particular needs of a younger clientele, both medical and psychological, can be met by the same program (Kim et al. 2007). 